\title{
Successful testing of the LISA Technology Package (LTP) interferometer engineering model
}

\author{
G Heinzel $^{1}$, C Braxmaier ${ }^{2}$, M Caldwell $^{3}$, K Danzmann $^{1,4}$, F Draaisma $^{5}$,

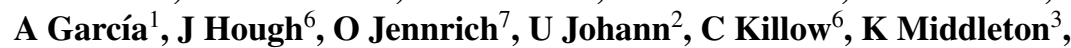

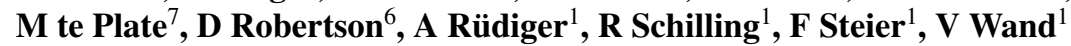 \\ and $\mathrm{H}$ Ward $^{6}$ \\ ${ }^{1}$ Max-Planck-Institut für Gravitationsphysik (Albert-Einstein-Institut), Callinstrasse 38, \\ D-30167 Hannover, Germany \\ 2 EADS Astrium GmbH, 88039 Friedrichshafen, Germany \\ ${ }^{3}$ Rutherford Appleton Laboratories, Chilton, UK \\ ${ }^{4}$ Universität Hannover, Institut für Atom- und Molekülphysik, Callinstr. 38, D-30167 Hannover, \\ Germany \\ 5 The Netherlands Organisation for Applied Scientific Research (TNO), Technisch Physische \\ Dienst (TPD), Delft, The Netherlands \\ ${ }^{6}$ Department of Physics and Astronomy, University of Glasgow, Glasgow, UK \\ ${ }^{7}$ ESTEC, Noordwijk, The Netherlands \\ E-mail: gerhard.heinzel@aei.mpg.de
}

Received 8 November 2004, in final form 10 January 2005

Published 21 April 2005

Online at stacks.iop.org/CQG/22/S149

\begin{abstract}
The LISA Technology Package (LTP), to be launched by ESA in 2008, is a technology demonstration mission in preparation for the LISA spaceborne gravitational wave detector. A central part of the LTP is the optical metrology package (heterodyne interferometer with phasemeter) that measures the distance between two test masses with a noise level of $10 \mathrm{pm} \mathrm{Hz}^{-1 / 2}$ between $3 \mathrm{mHz}$ and $30 \mathrm{mHz}$ and also the test mass alignment with a noise level of $<10 \mathrm{nrad} \mathrm{Hz}^{-1 / 2}$. An engineering model of the interferometer has been built and environmentally tested. Extensive functionality and performance tests were conducted. This paper reports on the successful test results.
\end{abstract}

PACS numbers: 06.30.Bp, 06.30.Gv, 07.60.Ly, 07.87.+v, 42.30.Rx, 95.55.Ym (Some figures in this article are in colour only in the electronic version)

\section{Introduction}

The LISA Technology Package (LTP) aboard the LISA Pathfinder (LPF) mission is an important milestone towards the LISA mission. Its purpose is to demonstrate the operation of free-floating test masses as mirrors of an interferometer and the performance of the drag-free 
operation. The sensitivity goal is relaxed by a factor of 10 with respect to the LISA specifications and thus is $3 \times 10^{-14} \mathrm{~m} \mathrm{~s}^{-2} \mathrm{~Hz}^{-1 / 2}$ at $1 \mathrm{mHz}$ for LPF.

The interferometer of the LTP continuously monitors the test masses in all operating modes by measuring

- the distance between the two test masses (called $x_{1}-x_{2}$ ),

- the position of one test mass with respect to the optical bench (called $x_{1}$ ),

- the differential alignment of the two test masses (with two sets of measurements: dc and differential wavefront sensing [2]),

- the alignment of one test mass with respect to the optical bench.

These signals not only serve as diagnostic monitor, but also as feedback signals for test mass stabilization in some operational modes.

The principle of operation and general design of the interferometer and phasemeter are described in $[1,2]$. An engineering model (EM) of the interferometer has been constructed using the same optical design, materials and bonding techniques that are planned for the flight model (FM). The EM was subjected to comprehensive environmental tests, which it survived without any measurable degradation or change. The functionality, performance and noise level of the interferometric measurement system were characterized. This paper describes the construction techniques of the optical bench (OB), the environmental tests and the measured performance which is very satisfactory overall and within the specifications at almost all frequencies.

\section{Construction}

The optical bench was manufactured under EADS Astrium lead at Rutherford Appleton Laboratories (RAL) according to the optical design given in [1,2] with support from Glasgow University and AEI Hannover. The baseplate is made from Zerodur ${ }^{\mathrm{TM}}$, and the optical components (of typical size $7 \mathrm{~mm} \times 15 \mathrm{~mm} \times 20 \mathrm{~mm}$ ) were attached by hydroxy-catalysis bonding $[3,4]$ to form a very rugged quasi-monolithic structure. Most of the components were aligned using a template manufactured from brass. The template was manufactured to approximately $10 \mu \mathrm{m}$ accuracy, and the resulting absolute positioning accuracy of the components was estimated to be approximately $0.5 \mathrm{~mm}$. The final recombination beamsplitters were aligned with light beams present and photodiodes installed by observing and optimizing the photodiode signals (interference contrast and mutual beam alignment) in real time. The beamsplitters were pre-aligned using a mechanical manipulator and fine aligned during the few minutes before bonding sets in after application of the bonding agent.

A handling accident in a late assembly stage caused four components that were already bonded to break. Those bonds older than a few weeks broke in the glass, not at the bond. This involuntarily demonstrated the strength of the quasi-monolithic bond. Furthermore, it forced us to develop a repair strategy which consisted in bonding small interface plates (formed like a bridge) over the damaged part of the baseplate and then bonding a new component onto the bridge plate (see figure 1, right).

In the next assembly stage, sideplates and stiffening rods made from Zerodur ${ }^{\mathrm{TM}}$ (see figure 1 , left) were attached by EADS Astrium Immenstaad using the 'insert' technology to reliably connect Zerodur $^{\mathrm{TM}}$ pieces to each other.

\section{Environmental testing}

The optical bench EM was subjected to thermo-vacuum and vibrational testing at the facilities of TNO/TPD Delft with personnel from TNO/TPD, AEI Hannover and EADS Astrium 

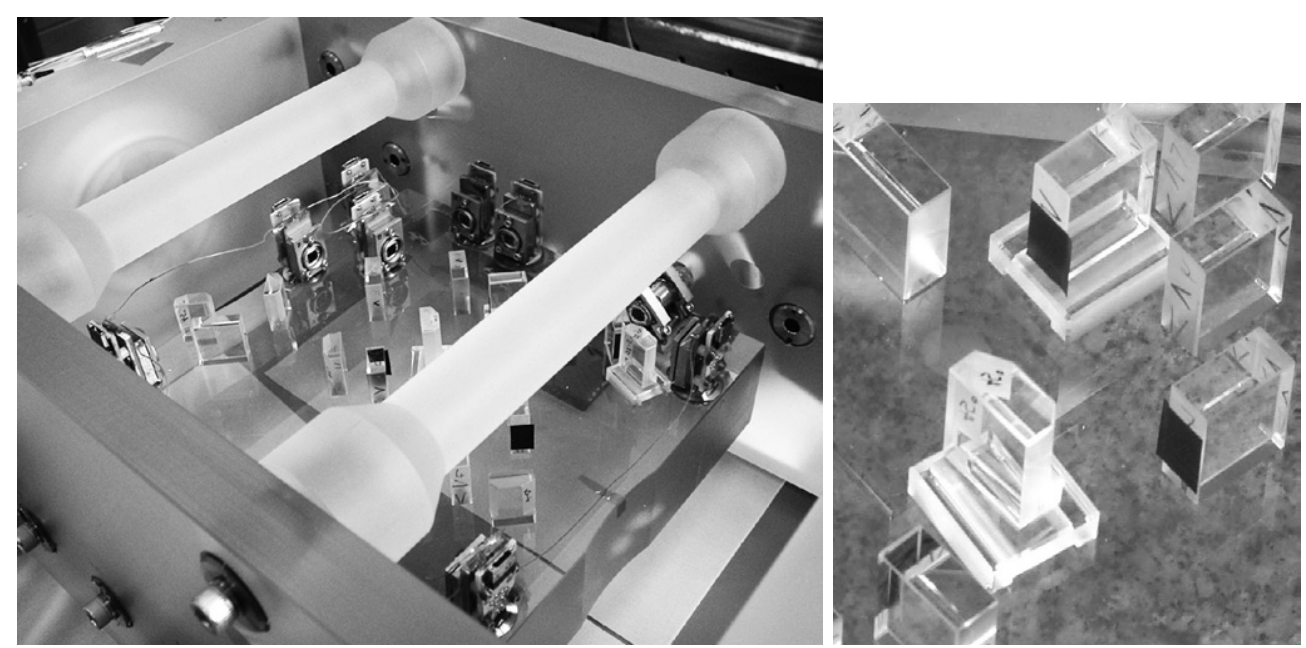

Figure 1. Picture of the optical bench (OB) engineering model (EM) and detail of the bridge plates used to repair broken bonds.

Immenstaad during the first half of 2004 [5]. Dummy masses having the same mass and moments of inertia as the real test mass enclosures were fixed to the Zerodur ${ }^{\mathrm{TM}}{ }^{\text {structure. }}$ Thermal cycling included several cycles between $0{ }^{\circ} \mathrm{C}$ and $40{ }^{\circ} \mathrm{C}$, and the vibrational tests included sine-wave and random excitations in three axes reaching up to $25 \mathrm{~g}$ at the struts.

A comparison of the alignment data of each photodiode recorded before and after the environmental tests yielded the following upper limits:

- No beam position has moved more than $5 \mu \mathrm{m}$ on any photodiode.

- No beam injector has changed in alignment by more than $20 \mu \mathrm{rad}$.

These changes, if at all real, are of no significance to the functionality and performance of the optical bench. They also imply that none of the bonded components have moved.

\section{Functional tests}

A comprehensive set of functional and performance tests was carried out while the optical bench EM was mounted in a large vacuum chamber at TNO/TPD. The tests were carried out with personnel and equipment from AEI Hannover, in particular the laser, modulation bench and phasemeter that were used in the prototype development. Functionally, the interferometer behaved completely according to the predictions, in particular:

- The test mass displacement fluctuations $x_{1}$ and $x_{1}-x_{2}$ can be measured with constant high sensitivity at any arbitrary displacement of the test masses along the sensitive $x$-axes within several $100 \mu \mathrm{m}$.

- The differential wavefront sensing (DWS) alignment measurements yield very sensitive alignment information for both test masses if each test mass is within $\pm 500 \mu \mathrm{rad}$ of its nominal position.

- For misalignments of a test mass larger than $\pm 500 \mu \mathrm{rad}$, the interferometer contrast is lost. The (less sensitive) dc alignment signals, however, still yield useful information about the direction of the misalignment. 

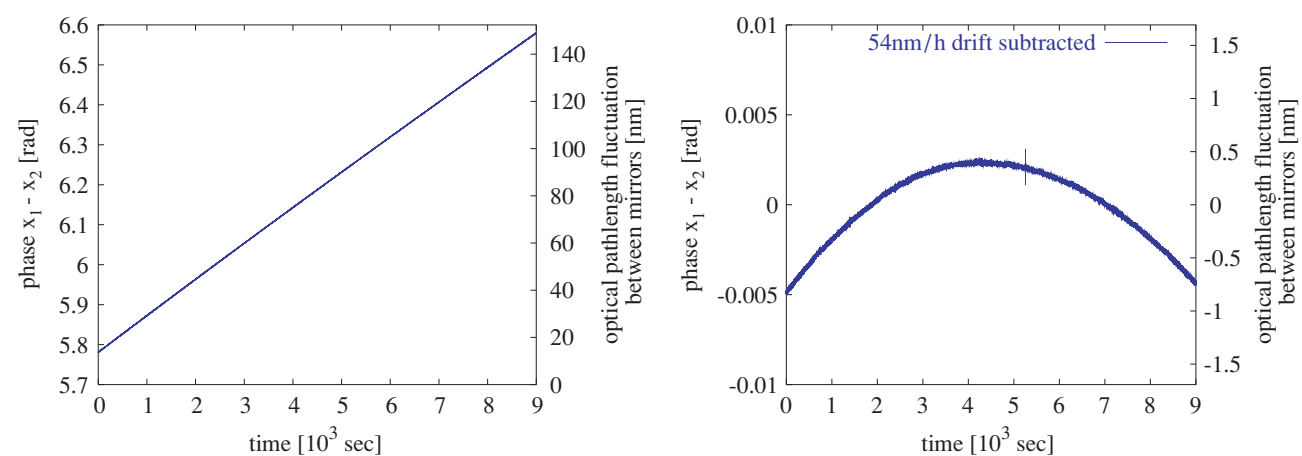

Figure 2. Time series of the $x_{1}-x_{2}$ measurement. In the right-hand graph, a best-fit linear function was subtracted.

- All these measurements produce valid results as soon as properly modulated light arrives at the optical bench and the phasemeter is operational. No acquisition or 'locking' procedure is necessary. The interferometer recovers immediately and automatically from momentary dropouts of, e.g., the laser power or blocking of a beam.

\section{Performance tests}

The purpose of the performance tests was to demonstrate the noise behaviour of the interferometer. Out of several stretches of data taken overnight, one stretch of $9000 \mathrm{~s}$ length was selected that showed little external disturbance. The contrast of all interferometers was stable between 80 and $90 \%$.

Figure 2 shows a typical time series of the $x_{1}-x_{2}$ measurement. It is dominated by a linear thermal drift. If this drift is subtracted (right picture), it is still dominated by a slow drifting motion. These drifts are probably due to thermal effects in the metal mirror mounts. The interferometer noise is barely visible as the finite thickness of the line in the right-hand graph.

Figure 3 shows a typical linear spectral density of the $x_{1}-x_{2}$ measurement. The dark solid curve shows the measurement described here. For comparison, several other curves are also shown (from top to bottom at $2 \times 10^{-2} \mathrm{~Hz}$ ):

- A curve measured at TNO in a comparable configuration, but without the fibre optical pathlength (OPD) stabilization (see [6]). In all three measurements below, OPD stabilization was used.

- The LPF mission goal. Note that in contrast to earlier inconsistent interpretations, the $y$-axis is now consistently referring to optical pathlength, and the LPF mission goal of $85 \mathrm{pm} \mathrm{Hz}^{-1 / 2}$ test mass displacement is hence plotted as $170 \mathrm{pm} \mathrm{Hz}^{-1}$ optical pathlength (at $30 \mathrm{mHz}$ ).

- The earlier 'best curve' with quadrant diodes [6], from the Glasgow prototype interferometer (also developed during the pre-investigations), measured in a collaboration between staff from Glasgow and AEI at Glasgow University using both Glasgow and AEI equipment.

- The LPF interferometer goal. This is unchanged by the re-interpretation and remains at $9 \mathrm{pm} \mathrm{Hz}^{-1 / 2}$ optical pathlength (at $30 \mathrm{mHz}$ ). 


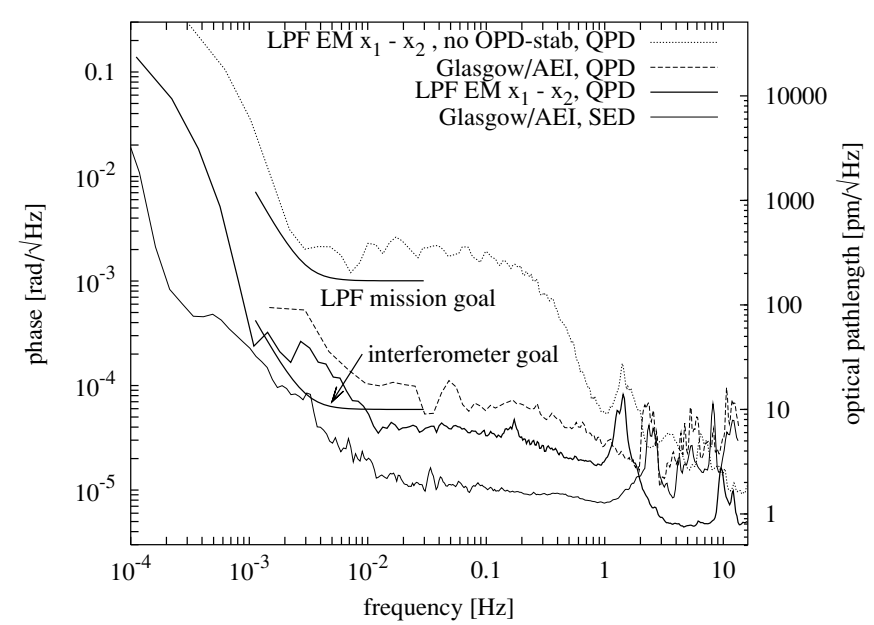

Figure 3. Linear spectral density of the $x_{1}-x_{2}$ measurement (see the text). 'QPD' and 'SED' refer to quadrant- and single-element diodes, respectively.

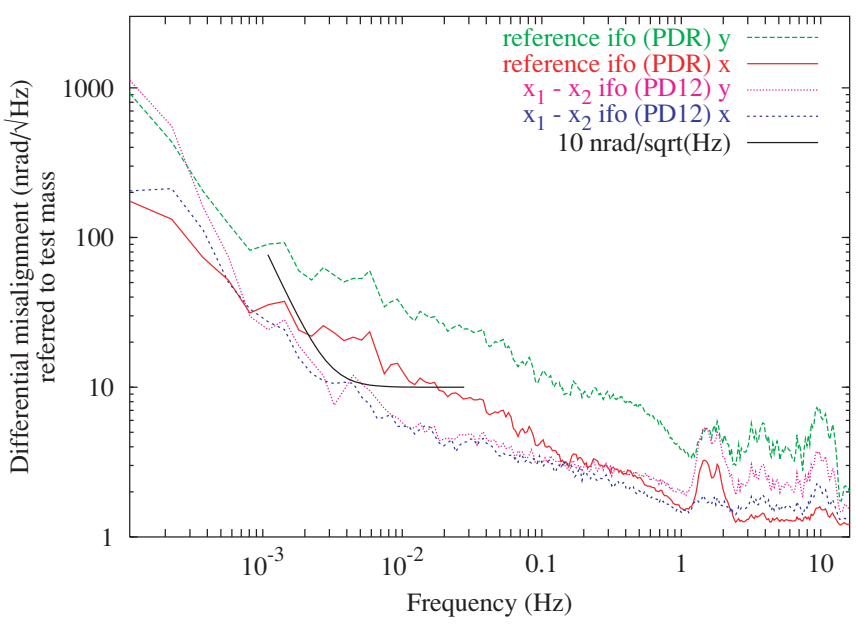

Figure 4. Linear spectral density of the DWS alignment measurements (see the text).

- The result discussed here, which represents a new 'best curve' as measured with quadrant diodes.

- The 'best curve', from the Glasgow prototype interferometer (also developed during the pre-investigations), measured in a collaboration between staff from Glasgow and AEI at Glasgow University using both Glasgow and AEI equipment [6]. This curve was measured with single-element diodes, which consistently show a better noise behaviour than quadrant diodes, the reason for which is under investigation.

Figure 4 shows a linear spectral density of the DWS alignment measurements.

The predicted noise level of $10 \mathrm{nrad} \mathrm{Hz}^{-1 / 2}$ (referred to the test mass misalignment) is reached in the $x_{1}-x_{2}$ measurement, where the static misalignment could be minimized. The higher noise of the reference interferometer (photodiode PDR) alignment can be traced to the fact that this diode shows a large static DWS misalignment (due to the imperfect beam injector alignment). 


\section{Conclusions}

The interferometry for LISA itself presents additional challenges, in particular very low received light power, Doppler shifts of up to $10 \mathrm{MHz}$, a very tricky alignment acquisition procedure and a much more severe influence of laser phase noise. Hence the LPF interferometry concept is not directly applicable for LISA. However, apart from being an essential tool for the verification of the drag-free control aboard LISA pathfinder, the LPF interferometer does nevertheless represent a significant step forward for the LISA interferometry, since the basic construction techniques that have been developed here can also be applied, their stability has been demonstrated and valuable experience has been gained.

\section{Acknowledgments}

The authors acknowledge support from the ESA/ESTEC 'Optical bench' contract, PPARC, the University of Glasgow and from DLR project 50OQ0002.

\section{References}

[1] Heinzel G et al 2003 Interferometry for the LISA Technology Package (LTP) aboard SMART-2 Class. Quantum Grav. 20 S153-61

[2] Heinzel G et al 2004 The LTP interferometer and phasemeter Class. Quantum Grav. 21 S581-7

[3] Gwo D-H 2001 Stanford University US patent no 6,284,085 B1

[4] Sneddon P et al 2003 Class. Quantum Grav. 20 5025-37

[5] Braxmaier C et al 2004 LISA Pathfinder optical interferometry Proc. SPIE 5500 164-73

[6] Robertson D 2005 LTP Interferometer-noise sources and performance Class. Quantum Grav. 22 S155 